\title{
Articulatory Changes Following Treatment of Muscle Tension Dysphonia: Preliminary Acoustic Evidence
}

\author{
Christopher Dromey \\ dromey@byu.edu \\ Shawn L. Nissen \\ Shawn_Nissen@byu.edu \\ Ray M. Merrill \\ ray_merrill@byu.edu \\ Nelson Roy
}

Follow this and additional works at: https://scholarsarchive.byu.edu/facpub

Part of the Communication Sciences and Disorders Commons

\section{Original Publication Citation}

Dromey, C., Nissen, S. L., Roy, N., \& Merrill, R. M. (28). Articulatory changes following treatment of muscle tension dysphonia: Preliminary acoustic evidence. Journal of Speech, Language, and Hearing Research, 51, 196-28.

\section{BYU ScholarsArchive Citation}

Dromey, Christopher; Nissen, Shawn L.; Merrill, Ray M.; and Roy, Nelson, "Articulatory Changes Following Treatment of Muscle Tension Dysphonia: Preliminary Acoustic Evidence" (2008). Faculty Publications. 210.

https://scholarsarchive.byu.edu/facpub/210 accepted for inclusion in Faculty Publications by an authorized administrator of BYU ScholarsArchive. For more information, please contact ellen_amatangelo@byu.edu. 


\section{Articulatory Changes Following Treatment of Muscle Tension Dysphonia: Preliminary Acoustic Evidence}

\author{
Christopher Dromey \\ Brigham Young University, Provo, UT \\ Shawn L. Nissen \\ Brigham Young University \\ Nelson Roy \\ The University of Utah, Salt Lake City
}

Ray M. Merrill

Brigham Young University
Purpose: Primary muscle tension dysphonia (MTD), a voice disturbance that occurs in the absence of structural or neurological pathology, may respond to manual circumlaryngeal techniques, which ostensibly alter the posture of the larynx and/ or the configuration of the vocal folds without directly targeting supralaryngeal articulatory structures. Although the phonatory benefits of these techniques have been documented previously, this investigation examined whether acoustic evidence exists for articulatory changes accompanying successful management.

Method: In this retrospective study of a clinical database, pre- and post-treatment speech samples from 111 women with MTD were analyzed for acoustic evidence of supraglottal vocal tract changes associated with voice improvement, which was confirmed by perceptual ratings of dysphonia severity. The slopes of the first and second formants in diphthongs, as well as global measures of speech timing were acquired. Twenty younger females with normal voices were recorded twice, across a similar time-span to the disordered speakers, to allow comparisons in performance. Results: Repeated measures analysis of variance was used to evaluate changes accompanying treatment. Significant time by group interactions for /al/ F2 slope, /el/ F2 slope, sample duration, and speaking time ratio were observed. As compared to the controls, diphthong second formant transitions increased in slope, and timing measures showed increases in speech continuity for the speakers with MTD. Conclusions: Collectively, these preliminary findings suggest that individuals with MTD experience changes in both articulatory and phonatory behavior following successful treatment that targets the larynx.

KEY WORDS: muscle tension dysphonia, articulatory acoustics, treatment effects

$\mathrm{n}$ its purest form, primary muscle tension dysphonia (MTD) is a voice disturbance that occurs in the absence of structural or neural pathology and may account for $10 \%-40 \%$ of cases referred to multidisciplinary voice clinics (Bridger \& Epstein, 1983; Koufman \& Blalock, 1991; Sama, Carding, \& Price, 2001; Schalen \& Andersson, 1992). Excessive or poorly regulated activity of the intrinsic and extrinsic laryngeal muscles is often cited as the proximal cause of MTD, but the source of this atypical muscle activity remains unclear. In this regard, MTD has been attributed to a variety of potentially partially overlapping sources, including (a) technical misuses of the vocal mechanism in the context of extraordinary voice demands (Koufman \& Blalock, 1988; Morrison, Nichol, \& Rammage, 1986; Morrison \& Rammage, 1993; Morrison, Rammage, Gilles, Pullan, \& Nichol, 1983), (b) learned adaptations following upper respiratory 
tract infection (Koufman \& Blalock, 1982; Milutinovic, 1991), (c) increased muscle tone secondary to the laryngopharyngeal reflux reflex (Morrison, 1997), (d) psychological and/or personality factors that tend to induce elevated tension in the laryngeal region (Aronson, 1990; Butcher, 1995; Butcher, Elias, \& Raven, 1993; Rammage, Nichol, \& Morrison, 1987; Roy \& Bless, 2000), and (e) extreme compensation for minor glottic insufficiency and/or underlying mucosal disease, which in clinical circles is sometimes referred to as secondary MTD.

Despite uncertainty surrounding its causal mechanisms, the clinical voice literature is replete with evidence that symptomatic voice therapy for primary MTD can often result in rapid and dramatic voice improvement (Andersson \& Schalen, 1998; Aronson, 1990; Carding \& Horsley, 1992; Carding, Horsley, \& Docherty, 1999; Koufman \& Blalock, 1988; Pannbacker, 1998; Ramig \& Verdolini, 1998; Roy \& Bless, 1998; Roy, Bless, Heisey, \& Ford, 1997; Roy \& Leeper, 1993). Because laryngeal hyperfunction is frequently cited as the principal cause of MTD, many voice therapies-including yawn-sigh, resonant voice therapy, visual and electromyographic biofeedback, progressive relaxation, and circumlaryngeal massage - aim to reduce or rebalance such laryngeal tension (Aronson, 1990; Boone \& McFarlane, 2000; Ramig \& Verdolini, 1998). Prolonged hypercontraction of extrinsic laryngeal muscles, especially the suprahyoids, is said to be associated with elevation of the larynx and hyoid bone and with associated pain and discomfort when the circumlaryngeal region is palpated (Lieberman, 1998; Roy \& Bless, 1998; Roy et al., 1997; Rubin, Lieberman, \& Harris, 2000). Furthermore, several voice clinicians have described manual/digital techniques to determine the presence and degree of laryngeal musculoskeletal tension as well as methods to improve such tension during the diagnostic assessment and management session (Aronson, 1990; Lieberman, 1998; Peifang, 1991; Roy \& Bless, 1998). These manual laryngeal techniques, including circumlaryngeal massage, have been shown to be particularly effective (Roy et al., 1997; Roy, Ford, \& Bless, 1996; Roy $\&$ Leeper, 1993). The hypothesized physical effect of such massage is reduced laryngeal height and stiffness along with increased mobility. Once laryngeal tension is normalized and range of motion is restored, an improvement in vocal effort, quality, and dynamic range reportedly follows.

Given the position of the larynx in the neck and the tendency for individuals with MTD to apparently experience elevated laryngeal postures, it is reasonable to anticipate abnormally high suprahyoid muscle activity in these speakers (Redenbaugh \& Reich, 1989). Indeed, clinical reports confirm that successful treatment of MTD can often be achieved by reposturing the hyoid, from which the larynx is suspended (Morrison \& Rammage, 1994; Roy \& Bless, 1998; Rubin et al., 2000). Moreover, muscles from the tongue and mandible attach to the hyoid bone from above and in front, and the hyoid bone forms the inferior attachment for the bulk of the tongue musculature. All of these suprahyoid muscles - including the digastrics (anterior and posterior), mylohyoid, geniohyoid, hyoglossus, and genioglossus, which attach to and suspend the hyoid - are referred to as the hyoid sling. We speculated, as others have (Morrison, 1997), that excessive tension within these suprahyoid muscles could reduce articulatory excursions because the tongue and/or jaw may be less free to move by virtue of their muscular attachments to the hyoid. If successful treatment of MTD reestablishes normal tension in the suprahyoid muscles, it would seem possible that lingual and mandibular activity could also be affected because of shared attachments with the hyoid bone, which putatively serves as a biomechanical link for the suprahyoid structures above and the larynx below. In this regard, Sapir (1989) noted that treatments for hyperfunctional voice often entail targeting extralaryngeal structures and "that articulatory movements may induce or exacerbate, via mechanical or neural coupling, the phonatory abnormalities" (p. 49). We wondered whether the reverse might also be true - that targeting hyolaryngeal structures might not only lead to phonatory improvements but also engender changes in supraglottal articulation. For instance, one possible mechanism might include circumlaryngeal massage, leading to reduced suprahyoid muscle activity, which then leads to depression of the hyoid and larynx, which then contributes to changes in jaw/tongue movements as well as vocal fold vibration, which ultimately lead to changes in acoustic variables during speech as well as phonatory improvements. Such depression of the hyoid and larynx could also contribute to an overall lowering of all formant frequencies because this would lengthen the vocal tract.

\section{Larynx-Articulation Interactions in Normal and Disordered Speakers}

Undoubtedly, many clinicians would view MTD as a disorder affecting the larynx only. This narrow focus, however, would overlook the findings from a number of studies that have documented coordination between laryngeal and supraglottal activity in both normal and disordered speech production, and the system-wide influence of "global" control variables such as loudness (Dromey \& Ramig, 1998). For example, when a healthy speaker increases vocal effort, the respiratory system must provide greater pressure to the larynx in addition to the changes in vocal fold configuration (Titze \& Sundberg, 1992). At the same time, there are increases in jaw opening, lingual excursions, and labial activity associated with louder speech (Dromey, 1995; Dromey \& Ramig, 1998; Schulman, 1989). 
In another example of possible laryngeal/ supralaryngeal coupling, the authors of one study found that vowel choice (/i/vs. /a/), which necessarily involves adjustments to tongue position, influenced electroglottographic, aerodynamic, and acoustic measures of laryngeal behavior (Higgins, Netsell, \& Schulte, 1998). They suggested that biomechanical effects, reflexive neural responses, and learned neural responses could underlie the observed changes in phonation. Others have documented the influence of jaw activity on measures of phonation. Cookman and Verdolini (1999) found that extreme jaw lowering led to increased vocal fold adduction, whereas reduced force in jaw closure was associated with lower levels of glottal adduction. These authors considered both biomechanical and neural bases as potential explanations for their findings. Postulating that articulatory movement speeds are directly related to neural drive, McClean and Tasko (2002) examined the correlations between jaw, lip, and tongue peak speeds and two key indices of vocal activity - fundamental frequency and intensity. Their findings led them to conclude that the neural control of laryngeal behavior is more closely coupled with mandibular activity than with the control of the lips and tongue. Davis and colleagues (Davis, Zhang, Winkworth, \& Bandler, 1996) also commented on the nature of respiratory, laryngeal, and oral articulatory coordination: "The fine and rapid changes in orofacial muscle activity associated with the production of speech consonants is highly coordinated with, but also independent of, the patterned laryngorespiratory activities" (p. 34). The findings of laryngeal-articulatory coordination in these basic science studies contributed, in part, to the motivation for the present work, as we sought to learn whether individuals with primary MTD might show evidence of altered supraglottal articulation following successful voice therapy.

In clinical circles, there have already been efforts to capitalize on these linkages by intensively targeting phonation in speakers with Parkinson disease (PD) while reaping articulatory benefits through a spreading of the therapy effects beyond the larynx (Dromey, 2000; Dromey, Ramig, \& Johnson, 1995; Kleinow, Smith, \& Ramig, 2001). In other words, treating the voice in PD automatically influences articulation because of the interdependence of the speech subsystems. Because biomechanical and/or neural coupling have been suggested as explanations for interactions between the voice and the articulators in healthy speakers, we reasoned that a condition involving increased laryngeal tension might be associated with altered oral articulatory behavior, if excessive tension is also present above the larynx. Certainly, previous work has documented unusual articulatory activity in spasmodic dysphonia (SD), a voice disorder that inexperienced clinicians often confuse with MTD because of shared auditoryperceptual features. In SD, kinematic investigations have revealed abnormal lip movements to co-occur with laryngeal spasms (Dromey, Reese, \& Howey, 2007; Tingley \& Dromey, 2000). Likewise, the presence of spasms prior to treatment has been associated with reduced ratings of speech fluency (Cannito et al., 1997), suggesting that the smooth flow of speech is interrupted by abnormal laryngeal activity. Although there is no reason to believe that MTD shares any etiological connection with either PD or SD, the published accounts which demonstrate that the activity of the larynx may influence the supraglottal vocal tract suggest that articulatory function might be linked to dysphonia in this condition. Furthermore, in an electromyographic study of MTD, Redenbaugh and Reich (1989) reported that excessive vocal fold adductory tension "may be accompanied by inappropriate activity in certain suprahyoid and infrahyoid muscles" (p. 68). Thus, an influence of MTD on lingual and/or mandibular activity would not be unanticipated, in addition to any potential raising of formant frequencies because of vocal tract shortening due to an elevated laryngeal posture.

\section{Acoustic Correlates of Articulatory Change: F2 Transition Slope and Speech Timing Measures}

In order to assess whether any acoustic evidence exists to support articulatory changes following treatment, it was necessary to identify and measure acoustic correlates of articulation. However, the retrospective design of the study necessarily limited the variables that were available for acoustic measurement. All of the speakers with MTD had read "The Rainbow Passage" (Fairbanks, 1960) before and after treatment, and the extraction of detailed articulatory acoustic measures was not anticipated at that time. Thus, one challenge was to find suitable targets in the recordings that would allow insights into vocal tract movements. At a minimum, preand post-therapy recordings would allow the generation of useful pilot data, and any patterns of change that might indicate vocal tract involvement would provide a useful starting point for the design of more targeted investigations in the future. Previous studies have examined diphthong second formant (F2) transitions in a number of populations. For example, flattened F2 transitions have been linked to reduced intelligibility in dysarthric speakers (Weismer, Martin, Kent, \& Kent, 1992) and have also been found to decrease following experimentally induced fatigue in individuals with normal speech (Solomon, 2000). In the present speakers, it was reasoned that formant changes during diphthongs would provide a useful window into vocal tract dynamics. Although the precise relationship between $\mathrm{F} 2$ and many articulatory parameters of the vocal tract remain ambiguous, a strong articulatory-acoustic association between 
F2 and some articulatory movements has been well established, such as tongue advancement and labial protrusion during vowel production (Ferrand, 2007; Kent \& Read, 2002; Rosner \& Pickering, 1994). This measure has been used in numerous studies of vocal tract behavior and was chosen in the present study because of the occurrence of several diphthongs in the recorded passage.

In the motor speech literature, reduced speech rate and the introduction of abnormal pauses have been noted in many individuals. Although speakers with MTD are not assumed to be dysarthric, it was reasoned that global measures of speech timing would be capable of detecting changes in vocal tract activity that might accompany changes in laryngeal function following treatment, if indeed the excessive laryngeal tension were to influence articulatory movements. Therefore, the sample duration and the relative proportion of speaking time during the sample were calculated as an index of speech continuity. If the strained phonation of MTD is linked to effortful vocal tract activity, it could be hypothesized that slow or discontinuous speech movements might result. Previous work has found increased segment durations and also higher listener ratings of disfluency in dysphonic speakers (Cannito et al., 1997; Cannito, Ege, Ahmed, \& Wagner, 1994). It was predicted, therefore, that if supralaryngeal vocal tract activity were to change following the treatment of disordered phonation, these timing measures would be sensitive to this change.

Thus, the present study was undertaken to explore the possibility that supraglottal articulation may change in speakers with primary MTD following successful voice treatment. The first and second formants in two diphthongs extracted from the reading passage were examined to measure the duration, extent, and slope of the transition. The duration of the reading passage and the proportion of time spent speaking were also measured. Listeners judged the voice quality to assess perceptual changes that would be reflective of treatment success. Analysis was conducted to compare values on these dependent measures before and after voice treatment was given. A group of control speakers read the same passage twice, with $1 \mathrm{hr}$ between recordings, to rule out the influence of practice effects in reading.

\section{$\overline{\text { Method }}$ \\ Participants}

The recordings in the study came from an archival database of pre- and post-treatment voice samples of 157 patients with MTD collected by the third author during routine clinical practice. Participants were diagnosed with pure MTD by a speech-language pathologist and an otolaryngologist, following extensive endoscopic and perceptual evaluation. Participants were included in the database based on their positive response to a single voice therapy session, conducted by the third author, who used manual circumlaryngeal techniques. Females in the database were then reviewed sequentially beginning with the first participant and going forward. Each participant who had a complete and analyzable dataset was selected for inclusion. Severity of dysphonia was not an inclusion or exclusion criterion. This approach generated a corpus of 111 women with MTD ( $M=46.12$ years, $S D=13.7$ ) who underwent manual circumlaryngeal therapy. The University of Utah Institutional Review Board approved the use of these voice samples and waived the requirement to obtain new consent from the participants. A detailed description of the assessment and treatment procedures is outlined in Roy and Bless (1998). Briefly, each participant underwent a case history, a traditional voice evaluation, and an assessment of musculoskeletal tension. Manual laryngeal reposturing maneuvers and/or circumlaryngeal massage were implemented to stimulate improved voice (Roy \& Bless, 1998; Roy et al., 1997). This approach does not deliberately attempt to target articulatory production or postures.

It was recognized that the second reading could be smoother than the first because of familiarity with the material and an increased level of comfort with the recording environment and that this could influence the dependent variables. The motivation for the inclusion of control speakers was to rule out the influence of a practice effect in reading. The retrospective and intraspeaker design of the study led to a decision to not attempt a close match of the disordered and control participants. The main intent was to uncover potential vocal tract changes that might accompany previously documented phonatory improvements (Roy et al., 1997; Roy \& Leeper, 1993) in the disordered speakers. Had a prospective study been undertaken, a closer matching of the demographic and physical characteristics of the two groups would have been sought. Because it was recognized that age might nevertheless exert an influence on the dependent measures, this variable was included as a covariate in the statistical analysis. Twenty women $(M=23.4$ years, $S D=7.0$ ) served as control speakers. They were recruited from the student population at Brigham Young University and had no reported history of speech, language, or hearing disorders.

\section{Task}

Before and after voice treatment, the speakers with MTD read the second and third sentences from "The Rainbow Passage" (Fairbanks, 1960) at a comfortable loudness level. Their speech was tape recorded and digitized offline at $25 \mathrm{kHz}$ with a Computerized Speech Lab system (Kay Elemetrics; Lincoln Park, NJ). The control speakers read the passage twice, with $1 \mathrm{hr}$ between 
readings to approximate the time difference between the pre- and post-treatment recordings for the disordered speakers. In the remainder of this report, the two recordings will be referred to as Time 1 and Time 2 for both sets of speakers.

\section{Experimental Design}

The main independent variable in this study was Time 1 versus Time 2 . The between-subjects factor used in statistical testing was the speaker group (MTD versus control). The dependent measures reflecting articulation were the transition extent, transition duration, and transition slope for F1 and F2 in the two diphthongs /eI/ and /aI/. Reading sample duration and speaking time ratio reflected longer-term temporal characteristics of speech during the reading task. Listener ratings of voice quality were used to assess perceptible changes from Time 1 to Time 2.

\section{Acoustic Analysis}

From the digital recordings, a number of acoustic measures were made from the two diphthongs (/eI, aI/) from the words rainbow, white, light, take, shape, high, and horizon in the reading passage (see Appendix). Frequency tracks for the first and second formants were extracted from these vowel targets using Praat acoustic analysis software, version 4.02 (Boersma \& Weenink, 1993). Specifically, a linear predictive coding (LPC)-based tracking algorithm (Burg method; 11 coefficients) was used to determine formant values for the vocalic segments at 5 -ms intervals. The LPC analysis used a 25-ms Hamming window with 50\% overlap and 98\% pre-emphasis. Each token was checked to ensure that surrounding speech sounds were not audible in the analyzed segment. In addition, these automatically tracked formants were visually inspected for accuracy and, where necessary, were hand-corrected prior to statistical analysis.

Using values from the extracted formant tracks, average $\mathrm{F} 1$ and $\mathrm{F} 2$ frequencies were calculated at eight different equidistant measurement points throughout each vowel's overall duration ( $\mathrm{t} 1-\mathrm{t} 8$ ). Thus, $\mathrm{t} 1$ was an average of the formant values in the initial $12.5 \%$ of the vowel's duration. Onset and offset values for the diphthongs were calculated at 25\% (t2-t3) and 75\% (t6-t7), respectively. It was reasoned that the influence of the consonantal context would be limited by computing the onset and offset at these points. The transition slope of the diphthongs was determined by the frequency difference between the onset and offset values as a function of time.

To evaluate the reliability of the extracted acoustic measures, speech samples from 10 speakers were randomly selected and reanalyzed by a second judge naive to the purpose of the experiment. This second set of duration and formant frequency measurements were extracted, recorded, and checked in the same manner as the original measures. Comparison of the two sets of duration measures produced a correlation of 0.96 , differing by an overall mean of approximately $2 \mathrm{~ms}$. Both the F1 and F2 frequency measures were correlated at 0.99 , with all correlations significant at $p<.001$. The mean absolute difference was $14.9 \mathrm{~Hz}$ for the $\mathrm{F} 1$ measures and $25.8 \mathrm{~Hz}$ for the $\mathrm{F} 2$ measures. It is important to note that differences in measures were distributed in a relatively even manner, with the overall mean difference being $<5 \mathrm{~Hz}$ for both the $\mathrm{F} 1$ and $\mathrm{F} 2$ measures.

\section{Passage Timing Measures}

The digital audio recordings were analyzed with a custom MATLAB (The MathWorks, 1994) routine that first created a root-mean-square (RMS) amplitude envelope of the entire spoken passage. It then set a threshold at $10 \%$ of the peak amplitude and detected upward or downward crossings of this threshold. When speech activity took the RMS values above this $10 \%$ threshold, the resulting segment was counted as speaking time, and values below the threshold were operationally defined as pauses. During the development of this algorithm, the threshold crossings were compared with the starting and ending points found when listening to the sample. The speaking and pause durations were found to be equivalent, and the algorithm was therefore deemed acceptable. The total sample duration was defined as the time from the first upward crossing of the threshold to the final downward crossing. A speaking time ratio was calculated by dividing the total duration of all spoken ( $>10 \%$ RMS threshold) segments divided by the total sample duration. These measures were made to allow an index of speech continuity because severely disordered phonation can result in breaks that listeners perceive as disfluent speech (Cannito et al., 1997).

\section{Perceptual Ratings}

Five young adults who were master's-level students in communication disorders served as listeners in a perceptual rating task. They listened over headphones in a quiet laboratory at a self-selected comfortable loudness level to the same Time 1 and Time 2 recordings that were analyzed for the acoustic and timing measures. The preand post-treatment recordings from the 111 speakers with MTD and the two sets of readings from the 20 control speakers were combined with an additional 38 randomly selected samples, which were repeated in order to test intrajudge reliability. All 300 samples (111 pre-treatment, 111 post-treatment for MTD, 20 first and 20 second readings for the controls, plus 38 repeats) were fully 
randomized, and each listener heard them in the same sequence. Using a custom MATLAB routine, the listeners used a computer mouse to move a slider on a screen to rate the voice quality. One end of the visual analog scale was labeled "normal," and the other end was labeled "profoundly abnormal." The position of the slider was stored by the software as a number that ranged from 0 to 100 , with higher numbers reflecting greater disorder severity. Intrarater reliability was assessed by calculating a Pearson correlation between the original and second ratings for the 38 repeated samples. The correlations ranged from .95 to $.98(M=.97)$ for the individual raters, and their judgments were thus deemed reliable. An intraclass correlation coefficient (ICC) was calculated to evaluate interrater reliability. This resulted in an average-measure ICC of .97 and a singlemeasure ICC of $.88, F(261,1044)=38.942, p<.001$, for both), indicating good consistency among the raters.

\section{Statistical Analysis}

As a test of the experimental hypothesis that voice improvements could influence articulatory acoustic measures, repeated measures analyses of variance (ANOVAs) were used to test Time 1 to Time 2 changes in the dependent measures. Group (MTD vs. control) was the betweensubjects factor, and age was included as a covariate because the two groups differed in their mean age. Wilks' Lambda $F$ value was used to assess the main Time 1 to Time 2 effect and the Time 1 to Time $2 \times$ Group interaction effect.

Because of the preliminary nature of the study and the goal of discerning in greater detail the patterns of change in the dataset, further exploratory testing was undertaken to provide more specific directions for future work in this area. Mean scores were derived for the dependent measures at Time 1 and Time 2, along with change scores, separately for the MTD and control groups. Distributions for these variables were sufficiently close to normality that the analyses were performed on untransformed data. The $t$ statistic was used to evaluate differences in means at Time 1, at Time 2, and in the mean of the change from Time 1 to Time 2 between MTD and control groups. It was also used to evaluate the mean of the change from Time 1 to Time 2 within groups. Tests of significance were evaluated against the null hypothesis of no difference, using the .05 level. Where variances differed between the two groups, the Satterthwaite test for unequal variances was used. Finally, Pearson and Spearman correlations were computed between voice severity ratings and the acoustic measures for all of the speakers at Time 1 as well as between changes in perceptual ratings and changes in Time 1 to Time 2 acoustic measures. Analyses were performed with standard packages of the Statistical Analysis System (SAS; SAS Institute, Cary, NC), Release 9.1 (SAS, 2003). Statistical assessment used the SAS procedures UNIVARIATE and GLM.

\section{$\overline{\text { Results }}$}

Means and standard deviations for each group in each condition are reported in Table 1 . The age distribution differed between the two groups: $M=46.2$ years, $S D=13.8$, for the speakers with MTD and $M=23.4, S D=$ 7.0 , for the controls, $t(50.6)=11.16, p<.0001$. For each of the dependent variables, a repeated measures ANOVA evaluated the overall time effect (Time 1 to Time 2) and the time effect in relation to group, age, and Age $\times$ Group. Age and Age $\times$ Group interaction were not significant in any of the models.

The repeated measures ANOVA identified significant overall time effects for speaking time ratio, $F(1)=$ $6.451, p=.012$, and perceptual severity, $F(1)=30.89$, $p<.0001$. No significant overall time effect appeared for the remaining variables. The Time $\times$ Group interaction effect revealed that the two groups changed differently with time for /aI/ F2 slope, $F(1)=4.036, p=.047 ; / \mathrm{eI} / \mathrm{F} 2$ slope, $F(1)=4.474, p=.036$; sample duration, $F(1)=4.276$, $p=.041$; speaking time ratio, $F(1)=6.483, p=.012$; and perceptual severity, $F(1)=64.970, p<.0001$. Mean values for the MTD and control participants' pre and post scores are shown for /aI/ F2 slope, /eI/ F2 slope, sample duration, and speaking time ratio (see Figure 1). The most marked difference between MTD and control group occur for the perceptual severity rating (see Figure 2).

As noted above, the second stage of statistical evaluation was exploratory in nature and went beyond the standard hypothesis-testing approach to examine patterns of change in each group from Time 1 to Time 2. A complete list of variable means at Time 1 and Time 2 as well as change scores from Time 1 to Time 2 is presented in Table 1. For the Time 1 recording, there were significant differences between the MTD and control groups for /aI/ F1 slope, /aI/ F1 transition extent, /eI/ transition duration, sample duration, speaking time ratio, and perceptual severity. For the Time 2 recording, there remained a significant difference between the MTD and control groups for the same variables. In addition, there was a significant difference between the groups for /eI/ F2 slope and /eI/ F2 transition extent.

Mean change was evaluated for each of the variables separately for MTD and control groups. Significant changes were observed for speakers in the MTD group for /aI/ F2 slope, /eI/ F1 slope, /eI/ F2 slope, /aI/ F2 transition extent, /eI/ F1 transition extent, /eI/ F2 transition extent, sample duration, speaking time ratio, and perceptual severity. For the control group, none of the change scores were significant. Significant differences (at the .1 level) in mean change scores between groups were 
Table 1. Means and standard deviations, and t test results for the speakers with muscle tension dysphonia (MTD) and controls.

\begin{tabular}{|c|c|c|c|c|c|c|c|c|}
\hline /al/ Fl slope (Hz/ms) MTD & 110 & -2.407 & 1.088 & -2.389 & 0.768 & 0.018 & 1.042 & 0.8557 \\
\hline Control & 20 & $-3.476^{+++}$ & 1.055 & $-3.420^{+++}$ & 0.859 & 0.056 & 0.700 & 0.7226 \\
\hline \multirow[t]{2}{*}{ /al/ F2 slope (Hz/ms) } & 110 & 6.047 & 1.579 & 6.676 & 1.522 & 0.629 & 1.576 & $<.0001$ \\
\hline & 20 & 6.340 & 1.597 & 6.064 & 1.485 & $-0.276^{+}$ & 1.151 & 0.2968 \\
\hline \multirow[t]{2}{*}{ /el/ F2 slope (Hz/ms) } & 110 & 2.728 & 1.304 & 3.156 & 1.250 & 0.428 & 1.642 & 0.0073 \\
\hline & 20 & 2.780 & 2.013 & $2.432^{+}$ & 2.311 & $-0.348^{\dagger}$ & 2.362 & 0.5178 \\
\hline \multirow[t]{2}{*}{ /al/ transition duration (ms) } & 110 & 82.8 & 18.7 & 81.6 & 14.6 & -1.2 & 14.4 & 0.3529 \\
\hline & 20 & 82.7 & 17.9 & 81.1 & 15.9 & -1.6 & 10.8 & 0.5412 \\
\hline \multirow[t]{2}{*}{ /al/ F1 transition extent $(\mathrm{Hz})$} & 110 & -191.915 & 75.172 & -190.058 & 62.872 & 1.857 & 77.559 & 0.8021 \\
\hline & 20 & $-248.300^{+}$ & 88.711 & $-236.000^{+}$ & 70.027 & 12.300 & 62.198 & 0.3875 \\
\hline \multirow[t]{2}{*}{ /el/ Fl transition extent $(\mathrm{Hz})$} & 110 & -45.924 & 40.575 & -56.118 & 35.958 & -10.194 & 48.077 & 0.0282 \\
\hline & 20 & -42.400 & 27.269 & -44.300 & 43.276 & -1.900 & 34.164 & 0.8062 \\
\hline \multirow[t]{2}{*}{ /el/ F2 transition extent $(\mathrm{Hz})$} & 110 & 180.756 & 84.179 & 208.839 & 84.205 & 28.083 & 100.803 & 0.0042 \\
\hline & 20 & 142.600 & 86.545 & $138.550^{+}$ & 81.197 & -4.050 & 70.839 & 0.8009 \\
\hline \multirow{2}{*}{ Sample duration (sec) } & 111 & 13.26 & 3.46 & 11.70 & 2.54 & -1.56 & 2.21 & $<.0001$ \\
\hline & 20 & $10.50^{++}$ & 0.90 & $10.29^{+}$ & 1.07 & $-0.21^{+}$ & 0.72 & 0.2056 \\
\hline \multirow[t]{2}{*}{ Speaking time ratio } & 111 & 0.838 & 0.113 & 0.901 & 0.055 & 0.063 & 0.105 & $<.0001$ \\
\hline & 20 & $0.924^{++}$ & 0.036 & $0.935^{+}$ & 0.024 & $0.011^{+}$ & 0.025 & 0.0616 \\
\hline \multirow[t]{2}{*}{ Perceptual severity } & 111 & 67.956 & 27.809 & 14.051 & 15.450 & -53.905 & 29.246 & $<.0001$ \\
\hline & 20 & $1.190^{+++}$ & 2.622 & $1.306^{+++}$ & 2.836 & $0.116^{+++}$ & 2.458 & 0.8344 \\
\hline
\end{tabular}

${ }^{*}$ Significant difference between MTD and control group in means at Time 1, Time 2, or in the change scores according to the $t$ statistic $\left({ }^{\dagger} p<.10,{ }^{+} p<.05\right.$, $\left.{ }^{++} p<.001,{ }^{+++} p<.0001\right) .{ }^{\dagger}$ Evaluating the mean change from Time 1 to Time 2 within groups.

observed for/aI/F2 slope, /eI/F2 slope, /aI/F2 transition extent, sample duration, speaking time ratio, and severity rating. With the exception of/aI/ F2 transition extent, which now showed a significant difference in change scores between groups, the results are consistent with the repeated measures analysis, in which age was included in the model.

There were several weak but statistically significant correlations between listener ratings of voice severity and the acoustic variables at Time 1 when speakers from both groups were combined. The coefficient and probability values are reported in Table 2 . Individuals whose voices were rated as higher in severity tended to have shallower F1 and F2 transition slopes for the /aI/ diphthong, which matched their smaller F1 and F2 transition extents for this sound. Higher dysphonia severity was associated with longer overall sample duration and lower speaking time ratio. Table 3 reports correlations between the change scores in perceptual severity and the changes in several of the acoustic variables. Speakers whose/aI/F2 slopes and transition extents increased more tended to have greater improvements in voice quality. In addition, the sample duration decreased and the speaking time ratio increased more for those speakers rated as having greater improvements in voice quality.

\section{$\overline{\text { Discussion }}$}

The present study was undertaken to learn whether vocal tract movements, as inferred from acoustic measures, change as a result of voice treatment in individuals with primary MTD. The Time $\times$ Group interaction effects from the repeated measures ANOVA confirm significant Time 1 to Time 2 speech acoustic changes, including an increase in F2 slope for the two diphthongs assessed, and fewer pauses along with overall shorter sample duration following treatment in the MTD group. There were clear post-treatment improvements in perceptual judgments of voice quality for these speakers. A group of control speakers who read the same material twice did not show similar changes in these measures, suggesting that the changes in the MTD group were not the result of a simple practice effect.

There is always a degree of ambiguity in the interpretation of articulatory acoustic data because of the 
Figure 1. Time 1 and Time 2 means and $95 \%$ confidence intervals for both speaker groups for the measures of diphthong second formant slopes, sample duration, and speaking time ratio.

/al/ F2 slope

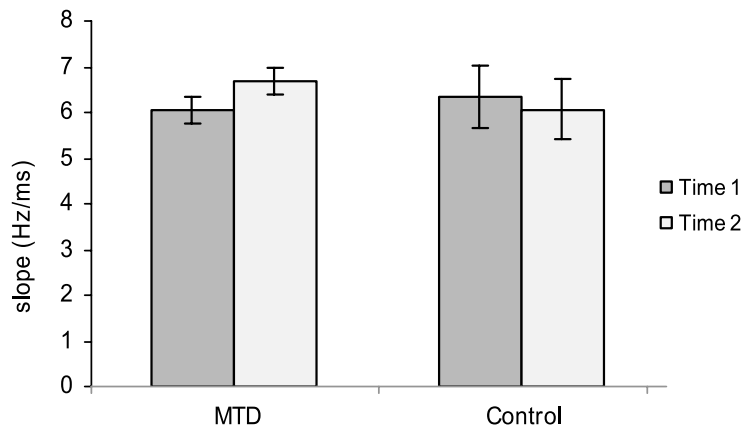

sample duration

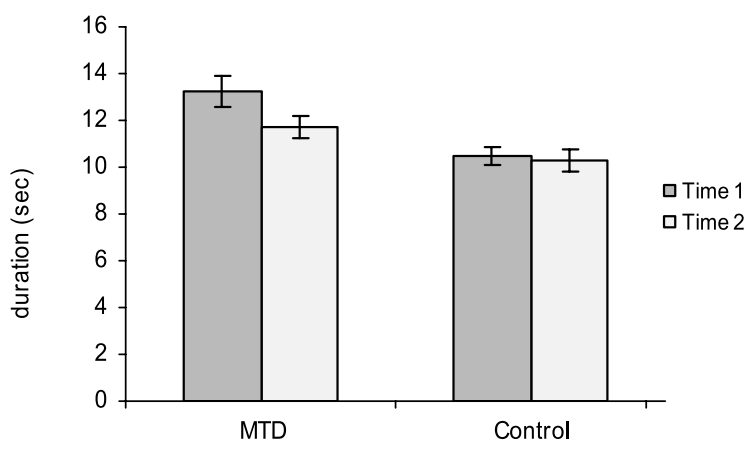

/el/ F2 slope

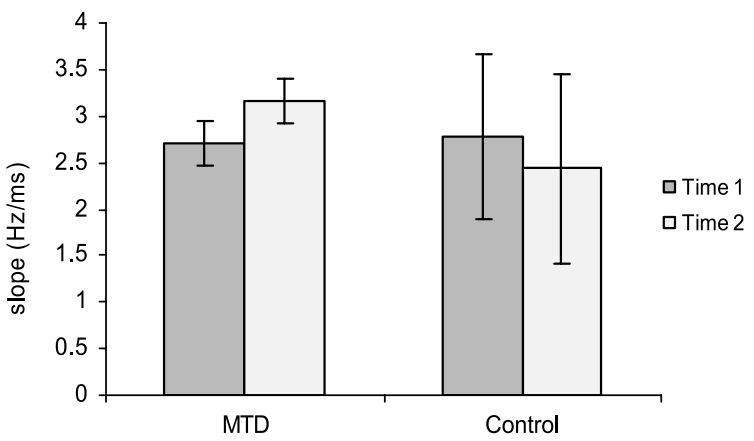

speaking time ratio

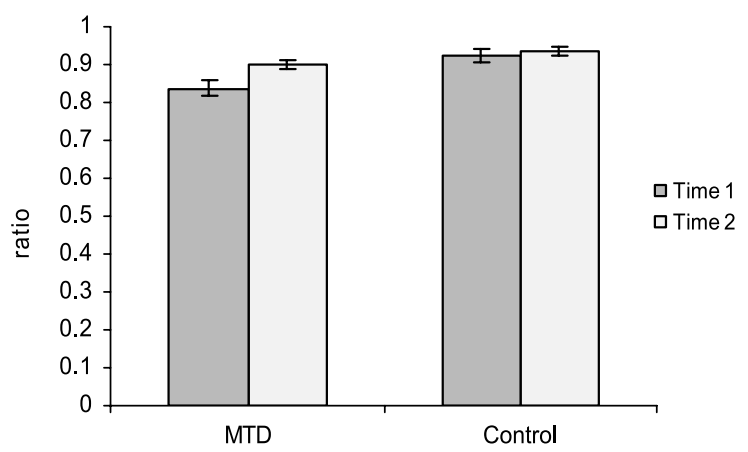

complex relationships between vocal tract activity and the way this activity is reflected in the acoustic signal. As a consequence, it is not possible to state with certainty which vocal tract structures contributed to the acoustic findings in the present study. However, based

Figure 2. Time 1 and Time 2 means and $95 \%$ confidence intervals for both speaker groups for the perceptual rating of severity.

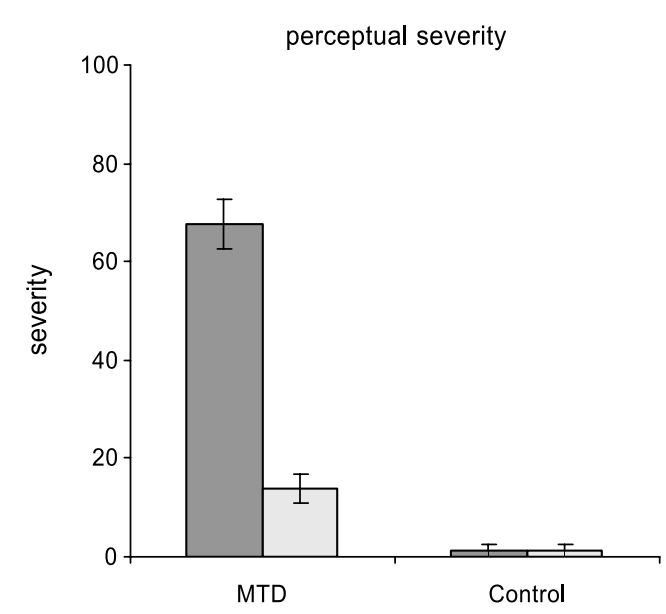

on the wide use of $\mathrm{F} 2$ slopes in the literature as an index of articulatory movement (Ferrand, 2007; Kent \& Read, 2002; Rosner \& Pickering, 1994), a number of interpretations can be offered and possible underlying mechanisms can be hypothesized.

Increased F2 slope values following treatment in the speakers with MTD reflect a more rapid change in

Table 2. Statistically significant correlations between perceived severity (higher numbers reflect greater severity) and acoustic speech measures in all of the speakers at Time 1.

\begin{tabular}{lrrrr}
\hline & $\begin{array}{c}\text { Pearson } \\
\text { correlation } \\
\text { coefficient }\end{array}$ & p value & $\begin{array}{c}\text { Spearman } \\
\text { correlation } \\
\text { coefficient }\end{array}$ & p value \\
\hline al F1 slope ${ }^{a}$ & 0.348 & $<.0001$ & 0.365 & $<.0001$ \\
al F2 slope & -0.315 & .0003 & -0.312 & .0003 \\
al F1 transition extent ${ }^{a}$ & 0.268 & .0021 & 0.225 & .0099 \\
al F2 transition extent & -0.247 & .0047 & -0.275 & .0015 \\
Sample duration & 0.418 & $<.0001$ & 0.496 & $<.0001$ \\
Speaking time ratio & -0.436 & $<.0001$ & -0.405 & $<.0001$ \\
\hline
\end{tabular}

${ }^{a} \mathrm{~F} 1$ slope and extent are negative numbers because the frequency decreases during /al/. 
Table 3. Statistically significant correlations between Time 1 and Time 2 change in perceived severity and between Time 1 and Time 2 change in acoustic variables for all of the speakers.

\begin{tabular}{lrrrr}
\hline \multicolumn{1}{c}{$\begin{array}{c}\text { Acoustic measure } \\
\text { change scores }\end{array}$} & $\begin{array}{c}\text { Pearson } \\
\text { correlation } \\
\text { coefficient }\end{array}$ & p value & $\begin{array}{c}\text { Spearman's } \\
\text { correlation } \\
\text { coefficient }\end{array}$ & p value \\
\hline /al/ F2 slope & 0.246 & .0049 & 0.240 & .0049 \\
/al/ F2 transition extent & 0.204 & .0199 & 0.221 & .0199 \\
Sample duration & 0.398 & $<.0001$ & 0.435 & $<.0001$ \\
Speaking time ratio & 0.292 & .0007 & 0.342 & $<.0001$ \\
\hline
\end{tabular}

frequency over time. The exploratory statistical evaluation of the components that make up the slope - the diphthong transition duration and transition extentrevealed that the extent of the F2 movement increased, but the duration did not change. Because the overall sample duration decreased for the speakers with MTD, the F2 slope theoretically might have increased by way of a decrease in duration for the same transition extent. However, this was not the case, and thus the slope increase cannot be attributed to adjustments in timing. Instead, the results would support the notion of increased vocal tract mobility after treatment of the voice. These findings are compatible with those reported in a case study of an individual with Parkinson disease who showed acoustic evidence of increased articulatory movements following intensive voice treatment (Dromey et al., 1995). Increases in this speaker's F2 transition extent were attributed to improved vocal tract dynamics that occurred without any clinical efforts to treat articulation but which arose instead from a system-wide increase in activity. In the current study, the voice treatment was focused on reducing laryngeal tension to improve vocal quality. In parallel with the Dromey et al. (1995) study, the perceptually obvious improvements in the voice were accompanied by formant changes that occurred without any of the speaker's effort being directed toward articulation. Although the F2 slope for both diphthongs increased following treatment for the speakers with MTD, the values for /eI/ were similar to those for the controls prior to treatment and were significantly larger in the post condition. On the other hand, the F2 slope for /aI/ did not differ from that of the controls for either reading, even though it changed significantly in the disordered speakers. Some of this apparent paradox may be due to the low statistical power associated with the small group of control speakers, whose voices were recorded to rule out practice effects in repeated readings and not to make detailed comparisons between the groups' speech performance at Time 1 and Time 2. Such findings point to the need for future prospective studies to involve larger groups of unimpaired speakers to allow clearer group comparisons in the performance of speech tasks selected for their suitability for articulatory acoustic analysis.
In the present study, the speakers with MTD may have had greater flexibility in oral articulatory movement following treatment. It could be speculated that this derives from biomechanical linkages between mandibular, lingual, and laryngeal muscles. According to this view, tightness in the larynx may lead to reduced flexibility in the movement of the hyoid, which then reduces the freedom with which the tongue and jaw may move. This view would be consistent with the mechanical explanations of articulator-larynx interactions suggested by previous authors (Higgins et al., 1998; McClean \& Tasko, 2002). An alternative explanation would be that individuals with MTD simultaneously experience excessive laryngeal and articulatory muscle tension, which both may respond to manual circumlaryngeal therapy techniques. When skillfully applied, systematic kneading and reposturing of the extralaryngeal region ostensibly stretch muscle tissue and fascia, promote local circulation with removal of metabolic wastes, and can relax tense muscles (Beck, 1994). In theory, this tension improvement could spread regionally, including to the suprahyoid musculature. However, if excessive neural drive to both laryngeal and articulatory muscles is responsible for tension in the articulators in MTD, the treatment would appear to have had an impact on the activity in both subsystems. Based on their study of unimpaired speakers, Cookman and Verdolini (1999) suggested that brain stem reflexes or cortical coactivation may be responsible for parallel increases in articulatory and laryngeal muscle contraction.

The acoustic data from the present study do not provide unequivocal evidence that the articulators moved more freely following voice treatment in MTD, and thus alternative explanations for the F2 slope findings must be considered. The relationship between the physical configuration of the vocal tract and the resulting formant frequency patterns is highly complex. Variations in a speaker's vowel formant frequencies are influenced by articulatory changes such as constriction size and location, mouth aperture, and transitory vocal tract length due to lip rounding (Rosner \& Pickering, 1994). However it is important to recognize that changes in F1 and F2 frequency can also be partially caused by momentary changes in the length and shape of the pharyngeal cavity. Laryngeal raising, which thereby shortens the overall resonating length of the posterior resonating cavity, can tend to increase the frequencies of the first and second formants. However, the relationship between the formant frequencies and the momentary length of the pharyngeal cavity is complicated by the possibility that speakers may compensate for the position of the larynx by modifying the shape of the cavity, thereby increasing or decreasing its overall volume and filtering characteristics (Rosner \& Pickering). In summary, it could be speculated that increasing the vocal tract length by lowering 
the larynx in these speakers might have contributed to complex formant interactions that would influence the F2 slope. Or, if the larynx were to become freer to move in the vertical dimension during speech following treatment, formant frequencies may undergo greater changes because of improved laryngeal flexibility. This uncertainty clearly points to the need for kinematic data in future investigations of possible articulatory changes in this population.

The reduction in overall sample duration and the increase in speaking time ratio in the speakers with MTD led them to be more similar to the controls than they had been prior to treatment. The changes in these variables suggest that speech became less fragmented or more continuous following treatment. This finding is consistent with results reported by Cannito and colleagues (Cannito, Woodson, Murry, \& Bender, 2004), whose listeners rated the speech of individuals with SD as more fluent following voice treatment. The present treatment was focused on the larynx, and these timing data suggest that the effects were more far-reaching than an improvement in voice quality. It could be speculated that phonation plays an important regulatory role in speech production in that fluent, continuous speech depends on reliable and predictable operation of the larynx (Dromey et al., 2007). Ramig (1992) noted that speech intelligibility can be compromised by dysphonia, and others have similarly documented the contribution of laryngeal function to speech intelligibility ratings (De Bodt, Hernandez-Diaz, \& Van de Heyning, 2002). Therefore, it appears reasonable that speech performance could have changed as laryngeal function became more normal in these speakers with MTD.

An alternative explanation for these timing findings could lie in the psychological state of the speakers. MTD often demands that individuals exert considerable effort to express themselves, which may lead to frustration during communication. The relief afforded by a reduction in laryngeal tension could have left these speakers more at ease following treatment and thus more able to read quickly and smoothly. Individuals arriving for assessment in the clinic may be more anxious at the start of the session than at the end, and these changes in their emotional state may also have contributed to the pre/post differences in speech timing measures, similar to the findings of an early study in which cognitive load was suggested to affect pause patterns (Goldman-Eisler, 1967).

The significant correlations between the perceptual ratings of voice quality and several of the acoustic variables provide an insight into the association between laryngeal and articulatory behavior in MTD. Although the correlations were significant, inferences must be drawn with caution because of the modest $r$ values. The listeners were only asked to rate voice quality, and no judgment was made of articulatory behavior or perceived intelligibility. Given this focus of the listeners, it is interesting that those speakers whose voices were judged to be more disordered in the initial recording were more likely to have shallower diphthong transition slopes with smaller frequency extents. This trend applied to both F1 and F2 for /aI/. These data lend support to the notion that the excessive tension that contributes to dysphonia may also underlie reduced articulatory flexibility. Longer sample durations and lower speaking time ratios tended to accompany greater dysphonia severity. It may be speculated that those speakers whose voices were more impaired might have experienced more speech hesitations, resulting in a longer overall duration, during which there was proportionally less continuous speech. One possibility that must be acknowledged in this interpretation is that the listeners may have rated the voices as more severe because the speech was less continuous and that they were not reacting solely to the phonatory quality.

A number of limitations in the present study may provide useful directions for future work with this population. First, the study was retrospective, relying on existing clinical recordings of speakers with MTD. The nature of the recordings resulted in a limited set of tokens of each sound being available for analysis. A fully randomized study could allow individuals with MTD to be assigned to treated and untreated groups who produce a larger repertoire of phonetic samples that would allow for better averaging across multiple tokens. Further, a closer matching of disordered speakers with controls who are equivalent in age, height, weight, vital capacity, cardiovascular health, and other variables previously identified as potentially influencing phonatory function would place any articulatory acoustic findings in a context that more readily lends itself to clearer interpretation. The ambiguity involved in drawing inferences about physiology from acoustic patterns could be overcome by collecting kinematic data from the tongue, lips, and jaw in this population. In particular, because F2 can be influenced by lingual and mandibular contributions, tracking the movements of these articulators would add valuably to our understanding of the impact of voice treatment on vocal tract function. In addition, electromyographic recordings of laryngeal and supraglottic vocal tract muscles would provide insights into possible mechanisms behind any treatment-related changes in vocal tract activity.

\section{Conclusions}

Regardless of the exact mechanism of pre/post change, the present results have shed valuable light on the interconnected nature of laryngeal and articulatory activity in a large clinical sample. The data suggest that the 
impact of a voice disorder may extend beyond the larynx and that, for this population at least, the communication disorder may be more of a speech production issue than solely a problem with the vocal folds. Whether the intervention has directly influenced supraglottic movements or has brought about positive change via more complex subsystem interactions, successful circumlaryngeal treatment of MTD has been shown to influence acoustic measures of supraglottal activity and not just the voice.

\section{Acknowledgment}

We express our appreciation to Kurtt Boucher for his assistance with the acoustical analysis.

\section{References}

Andersson, K., \& Schalen, L. (1998). Etiology and treatment of psychogenic voice disorder: Results of a follow-up study of thirty patients. Journal of Voice, 12, 69-106.

Aronson, A. E. (1990). Clinical voice disorders: An interdisciplinary approach (3rd ed.). New York: Thieme.

Beck, M. F. (1994). Theory and practice of therapeutic massage (2nd ed.). Albany, NY: Milady Publishing Company.

Boersma, P., \& Weenink, D. (1993). Praat (Version 4.02) [Computer software]. Amsterdam: Institute of Phonetic Sciences, the University of Amsterdam.

Boone, D. R., \& McFarlane, S. C. (2000). The voice and voice therapy (6th ed.). Boston: Allyn and Bacon.

Bridger, M. M., \& Epstein, R. (1983). Functional voice disorders: A review of 109 patients. Journal of Laryngology and Otology, 97, 1145-1148.

Butcher, P. (1995). Psychological processes in psychogenic voice disorder. European Journal of Disorders of Communication, 30, 467-474.

Butcher, P., Elias, A., \& Raven, R. (1993). Psychogenic voice disorders and cognitive behaviour therapy. San Diego, CA: Singular Publishing Group.

Cannito, M. P., Burch, A. R., Watts, C., Rappold, P. W., Hood, S. B., \& Sherrard, K. (1997). Disfluency in spasmodic dysphonia: A multivariate analysis. Journal of Speech, Language, and Hearing Research, 40, 627-641.

Cannito, M. P., Ege, P., Ahmed, F., \& Wagner, S. (1994). Diadochokinesis for complex trisyllables in individuals with spasmodic dysphonia and nondisabled subjects. In J. A. Till, K. M. Yorkston, \& D. R. Beukelman (Eds.), Motor speech disorders: Advances in assessment and treatment (pp. 91-100). Baltimore: Paul H. Brookes.

Cannito, M. P., Woodson, G. E., Murry, T., \& Bender, B. (2004). Perceptual analyses of spasmodic dysphonia before and after treatment. Archives of Otolaryngology-Head \& Neck Surgery, 130, 1393-1399.

Carding, P. N., \& Horsley, I. (1992). An evaluation study of voice therapy in non-organic dysphonia. European Journal of Disorders of Communication, 27, 137-158.
Carding, P. N., Horsley, I., \& Docherty, G. (1999). A study of the effectiveness of voice therapy in the treatment of 45 patients with nonorganic dysphonia. Journal of Voice, $13,72-104$.

Cookman, S., \& Verdolini, K. (1999). Interrelation of mandibular laryngeal functions. Journal of Voice, 13, 11-24.

Davis, P. J., Zhang, S. P., Winkworth, A. L., \& Bandler, R. (1996). Neural control of vocalization: Respiratory and emotional influences. Journal of Voice, 10, 23-38.

De Bodt, M. S., Hernandez-Diaz, H. M., \& Van de Heyning, P. H. (2002). Intelligibility as a linear combination of dimensions in dysarthric speech. Journal of Communication Disorders, 35, 283-292.

Dromey, C. (1995). Global and component-specific modifications of speech: A multi-level study. Boulder, CO: University of Colorado at Boulder.

Dromey, C. (2000). Articulatory kinematics in patients with Parkinson disease using different speech treatment approaches. Journal of Medical Speech-Language Pathology, 8, 155-161.

Dromey, C., \& Ramig, L. (1998). Intentional changes in sound pressure level and rate: Their impact on measures of respiration, phonation, and articulation. Journal of Speech, Language, and Hearing Research, 41, 1003-1018.

Dromey, C., Ramig, L. O., \& Johnson, A. B. (1995). Phonatory and articulatory changes associated with increased vocal intensity in Parkinson disease: A case study. Journal of Speech and Hearing Research, 38, 751-764.

Dromey, C., Reese, A., \& Howey, S. (2007). Lip kinematics in spasmodic dysphonia before and after treatment with botulinum toxin. Journal of Medical Speech-Language Pathology, 15, 263-277.

Fairbanks, G. (1960). Voice and articulation drillbook (2nd ed.). New York: Harper \& Row.

Ferrand, C. T. (2007). Speech science: An integrated approach to theory and clinical practice (2nd ed.). Boston: Allyn and Bacon.

Goldman-Eisler, F. (1967). Sequential temporal patterns and cognitive processes in speech. Language and Speech, 10, $122-132$.

Higgins, M. B., Netsell, R., \& Schulte, L. (1998). Vowelrelated differences in laryngeal articulatory and phonatory function. Journal of Speech, Language, and Hearing Research, 41, 712-724.

Kent, R. D., \& Read, C. (2002). The acoustic analysis of speech . Albany, NY: Delmar.

Kleinow, J., Smith, A., \& Ramig, L. O. (2001). Speech motor stability in IPD: Effects of rate and loudness manipulations. Journal of Speech, Language, and Hearing Research, 44, 1041-1051.

Koufman, J. A., \& Blalock, P. D. (1982). Classification and approach to patients with functional voice disorders. Annals of Otology, Rhinology, and Laryngology, 91, 372-377.

Koufman, J. A., \& Blalock, P. D. (1988). Vocal fatigue and dysphonia in the professional voice user: Bogart-Bacall syndrome. Laryngoscope, 98, 493-499.

Koufman, J. A., \& Blalock, P. D. (1991). Functional voice disorders. Otolaryngologic Clinics of North America, 4, 1059-1073. 
Lieberman, J. (1998). Principles and techniques of manual therapy: Application in the management of dysphonia. In T. Harris, S. Harris, J. S. Rubin, \& D. M. Howard (Eds.), The voice clinical handbook (pp. 91-138). London: Whurr Publishers.

McClean, M. D., \& Tasko, S. M. (2002). Association of orofacial with laryngeal and respiratory motor output during speech. Experimental Brain Research, 146, 481-489.

Milutinovic, Z. (1991). Inflammatory changes as a risk factor in the development of phononeurosis. Folia Phoniatrica, $43,177-180$.

Morrison, M. D. (1997). Pattern recognition in muscle misuse voice disorders: How I do it. Journal of Voice, 11, 108-114.

Morrison, M. D., Nichol, H., \& Rammage, L. A. (1986). Diagnostic criteria in functional dysphonia. Laryngoscope, $94,1-8$

Morrison, M. D., \& Rammage, L. A. (1993). Muscle misuse voice disorders: Description and classification. Acta OtoLaryngologica, 113, 428-434.

Morrison, M. D., \& Rammage, L. A. (1994). The management of voice disorders. San Diego, CA: Singular Publishing Group.

Morrison, M. D., Rammage, L. A., Gilles, M. B., Pullan, C. B., \& Nichol, H. (1983). Muscular tension dysphonia. Journal of Otolaryngology, 12, 302-306.

Pannbacker, M. (1998). Voice treatment techniques: A review and recommendations for outcome studies. American Journal of Speech-Language Pathology, 7, 49-64.

Peifang, C. (1991). Massage for the treatment of voice ailments. Journal of Traditional Chinese Medicine, 11, 209-215.

Ramig, L. A. (1992). The role of phonation in speech intelligibility: A review and preliminary data from patients with Parkinson's disease. In R. D. Kent (Ed.), Intelligibility in speech disorders: Theory, measurement and management (pp. 119-155). Amsterdam: John Benjamins.

Ramig, L., \& Verdolini, K. (1998). Treatment efficacy: Voice disorders. Journal of Speech, Language, and Hearing Research, 41, S101-S116.

Rammage, L. A., Nichol, H., \& Morrison, M. D. (1987). The psychopathology of voice disorders. Human Communication Canada, 11, 21-25.

Redenbaugh, M. A., \& Reich, A. R. (1989). Surface EMG and related measures in normal and vocally hyperfunctional speakers. Journal of Speech and Hearing Disorders, 54, 68-73.

Rosner, B. S., \& Pickering, J. B. (1994). Vowel perception and production. New York: Oxford University Press.

Roy, N., \& Bless, D. M. (1998). Manual circumlaryngeal techniques in the assessment and treatment of voice disorders. Current Opinion in Otolaryngology-Head and Neck Surgery, 6, 151-155.

Roy, N., \& Bless, D. M. (2000). Toward a theory of the dispositional bases of functional dysphonia and vocal nodules: Exploring the role of personality and emotional adjustment. In R. D. Kent \& M. J. Ball (Eds.), Voice quality measurement. San Diego, CA: Singular Publishing Group.
Roy, N., Bless, D. M., Heisey, D., \& Ford, C. N. (1997). Manual circumlaryngeal therapy for functional dysphonia: An evaluation of short- and long-term treatment outcomes. Journal of Voice, 11, 321-331.

Roy, N., Ford, C. N., \& Bless, D. M. (1996). Muscle tension dysphonia and spasmodic dysphonia: The role of manual laryngeal tension reduction in diagnosis and management. Annals of Otology, Rhinology, and Laryngology, 105, 851-856.

Roy, N., \& Leeper, H. A. (1993). Effects of the Manual Laryngeal Musculoskeletal Tension Reduction Technique as a Treatment for Functional Voice Disorders-Perceptual and Acoustic Measures. Journal of Voice, 7, 242-249.

Rubin, J. S., Lieberman, J., \& Harris, T. (2000). Laryngeal manipulation. Otolaryngologic Clinics of North America, 33, 1017-1034.

Sama, A., Carding, P. N., \& Price, S. (2001). The clinical features of functional dysphonia. Laryngoscope, 111, $458-463$.

Sapir, S. (1989). The intrinsic pitch of vowels: Theoretical, physiological, and clinical considerations. Journal of Voice, $3,44-51$.

SAS Institute. (2003). SAS (Version 9.1) [Computer software]. Cary, NC: Author.

Schalen, L., \& Andersson, K. (1992). Differential diagnosis and treatment of psychogenic voice disorder. Clinical Otolaryngology, 17, 225-230.

Schulman, R. (1989). Articulatory dynamics of loud and normal speech. The Journal of the Acoustical Society of America, 85, 295-312.

Solomon, N. P. (2000). Changes in normal speech after fatiguing the tongue. Journal of Speech, Langauge, and Hearing Research, 43, 1416-1428.

The MathWorks. (1994). MATLAB (Version 7.1) [Computer software]. Natick, MA: Author.

Tingley, S., \& Dromey, C. (2000). Phonatory-articulatory relationships: Do speakers with spasmodic dysphonia show aberrant lip kinematic profiles? Journal of Medical SpeechLanguage Pathology, 8, 249-252.

Titze, I. R., \& Sundberg, J. (1992). Vocal intensity in speakers and singers. The Journal of the Acoustical Society of America, 91, 2936-2946.

Weismer, G., Martin, R., Kent, R. D., \& Kent, J. F. (1992). Formant trajectory characteristics of males with amyotrophic lateral sclerosis. The Journal of the Acoustical Society of America, 91, 1085-1098.

Received May 19, 2006

Revision received September 29, 2006

Accepted June 6, 2007

DOI: 10.1044/1092-4388(2008/015)

Contact author: Christopher Dromey, 133 Taylor Building, Brigham Young University, Provo, UT 80602.

E-mail: dromey@byu.edu. 
Appendix. Sample from The Rainbow Passage (Fairbanks, 1960), showing the diphthong targets used in the articulatory acoustic analysis. The first number in each box is the number of the diphthong, and the second number in each box is the specific token of that sound.

The rainbow is a division of white light into many beautiful colors. These take the shape of a long round arch, with its path high above, and its two ends apparently beyond the horizon.

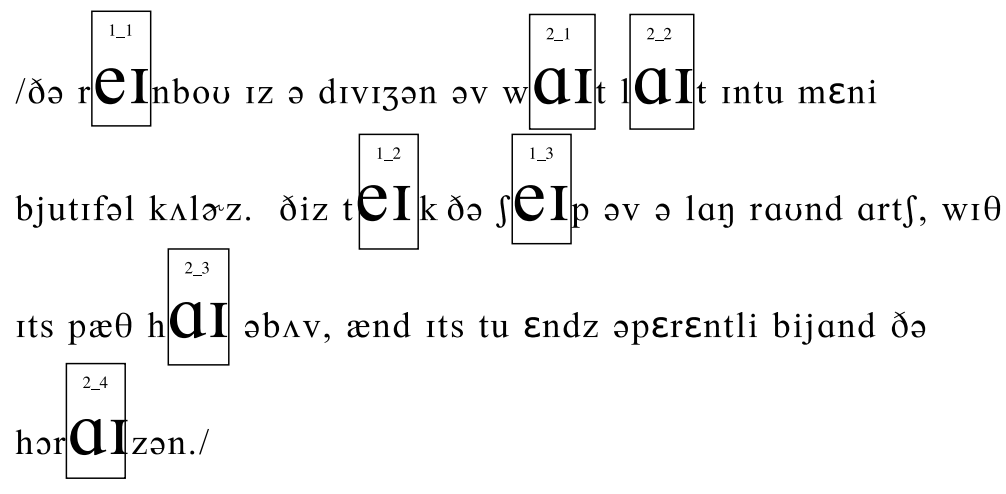

